
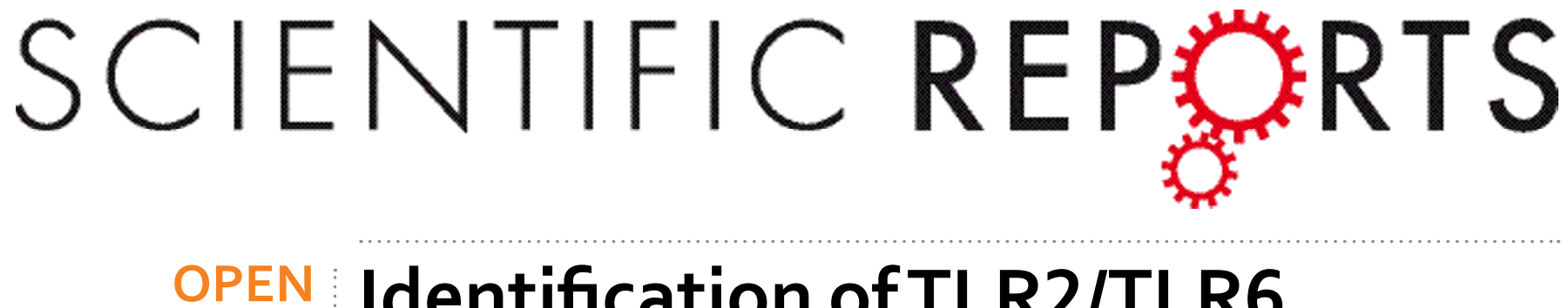

\title{
Identification of TLR2/TLR6 signalling lactic acid bacteria for supporting immune regulation
}

Received: 15 March 2016

Accepted: 13 September 2016

Published: 06 October 2016

\author{
Chengcheng Ren ${ }^{1,2}$, Qiuxiang Zhang ${ }^{2}$, Bart J. de Haan ${ }^{1}$, Hao Zhang $^{2}$, Marijke M. Faas ${ }^{1}$ \& \\ Paul de Vos $^{1}$
}

Although many lactic acid bacteria (LAB) influence the consumer's immune status it is not completely understood how this is established. Bacteria-host interactions between bacterial cell-wall components and toll-like receptors (TLRs) have been suggested to play an essential role. Here we investigated the interaction between LABs with reported health effects and TLRs. By using cell-lines expressing single or combination of TLRs, we show that LABs can signal via TLR-dependent and independent pathways. The strains only stimulated and did not inhibit TLRs. We found that several strains such as $L$. plantarum CCFM634, L. plantarum CCFM734, L. fermentum CCFM381, L. acidophilus CCFM137, and S. thermophilus CCFM218 stimulated TLR2/TLR6. TLR2/TLR6 is essential in immune regulatory processes and of interest for prevention of diseases. Specificity of the TLR2/TLR6 stimulation was confirmed with blocking antibodies. Immunomodulatory properties of LABs were also studied by assessing IL-10 and IL-6 secretion patterns in bacteria-stimulated THP1-derived macrophages, which confirmed species and strain specific effects of the LABs. With this study we provide novel insight in LAB specific host-microbe interactions. Our data demonstrates that interactions between pattern recognition receptors such as TLRs is species and strain specific and underpins the importance of selecting specific strains for promoting specific health effects.

Lactic acid bacteria (LAB) have been traditionally utilized for manufacturing fermented foods and are associated with a broad variety of beneficial health effects ${ }^{1}$. LAB also belong to the indigenous commensal microflora in humans and animals ${ }^{2}$. Some LAB strains have been confirmed to exert health-promoting effects in both animal studies and human clinical trials and are sometimes used as probiotics ${ }^{1}$. However, LAB strains from different species or even strains within the same species might possess totally different regulatory properties. Moreover, even though some LAB strains have been demonstrated to be beneficial for the remission of some diseases, they are not beneficial under all clinical circumstances ${ }^{3}$, and effects of some LABs are different in diseased and healthy individuals ${ }^{4,5}$. Therefore, it is imperative to select specific LAB strains for prevention or cure of specific disorders ${ }^{6}$.

Many LABs exhibit in vivo immunomodulatory properties by supporting intestinal immune cell responses ${ }^{7}$, reinforcing intestinal barrier function ${ }^{8}$, and inhibiting pathogenic adhesion ${ }^{9}$. Crosstalk between LAB and host cells in the gastrointestinal mucosa is a key determinant for modulatory activities ${ }^{10,11}$. Furthermore, recognition of LAB by the intestinal immune system is crucial for transducing their regulatory benefits to the host, which is initiated via the ligation of microbe-associated molecular patterns (MAMPs) exhibited on LAB to pattern recognition receptors (PRRs) expressed on immune cells and epithelial cells ${ }^{12}$. Toll-like receptors (TLRs) are the most important and best-characterized class of PRRs. At least nine human TLRs have been studied and recognize specific MAMPs on different microorganisms ${ }^{13}$.

TLRs play a cardinal role in recognizing microbial components by the innate immune system, which leads to downstream signalling cascades, inducing an immune response. Following binding of ligands to TLRs, adaptor molecules such as myeloid differentiation primary-response protein 88 (MyD88) will be recruited for transmitting downstream signalling responses. MyD88-dependent pathways comprise the vast majority of TLRs signalling including TLR2, 4, 5, 7, 8, and 9 although MyD88-independent pathways are indispensable for TLR3 signalling ${ }^{13}$. In relation to LABs, TLR2 and MAMPs have been studied in most detail. MAMPs on LAB cell-surfaces are peptidoglycan, lipoteichoic acid, and wall teichoic acid which are predominantly detected by TLR2 ${ }^{12}$. So far, a

${ }^{1}$ Immunoendocrinology, Division of Medical Biology, Department of Pathology and Medical Biology, University of Groningen and University Medical Center Groningen, Hanzeplein 1, 9700 RB Groningen, The Netherlands. ${ }^{2}$ School of Food Science and Technology, Jiangnan University, 1800 Lihu Road, Wuxi 214122, China. Correspondence and requests for materials should be addressed to Q.Z. (email: zhangqx@jiangnan.edu.cn) 
variety of studies have demonstrated that TLR2 signalling is a pivotal player in maintaining immune homeostasis especially in the intestine, which includes promoting repair of damaged epithelial mucosa, inhibiting intestinal inflammation and enhancing intestinal barrier integrity ${ }^{14-17}$. Dysregulation of TLR2 signalling is widely believed to be associated with a range of diseases such as autoimmune diseases and chronic inflammation ${ }^{18,19}$. This dual role is caused by the unique property of TLR2 signalling pathways that it can only be activated in the presence of TLR1 and TLR6 ${ }^{20,21}$. TLR2/TLR1 activation was shown to induce pro-inflammatory cytokines such as IL-17 and IL-12 while TLR2/TLR6 activation could elicit tolerogenic IL-10 secretion ${ }^{22-24}$.

It is worth noting that even though MAMPs on LAB share similar conserved basic structure, subtle structural distinctions of MAMPs among various LAB species and strains can result in activation of different TLRs pathways, thus resulting in differential modulatory properties ${ }^{25-27}$. We therefore screen in this study for all nine TLRs. Also costimulation of TLR2 and TLR6 was studied since TLR2 in combination with TLR6 triggers beneficial regulatory immune responses as described above. It is assumed that LAB strains stimulating TLR2/TLR6 pathways might have promising potentials for modulating excessive inflammatory reactions such as may occur in inflammatory bowel disease ${ }^{22}$. To identify whether LABs are capable of triggering TLR2/TLR6 signalling pathways, 16 different LAB strains of food and human origins were characterized and tested for their interactions with specific TLRs. The selection of these strains was based on a literature research for strains or parental strains that have reported health benefits ${ }^{28-35}$.

\section{Results}

Modulation of pro-and anti-inflammatory cytokines production in THP1 macrophages by different bacterial strains. A large number of species that potentially might serve as immune-active bacterial strains were selected from the Culture Collections of Food Microbiology $y^{28-35}$. In order to assess the immune-modulatory capacity of the various bacterial strains, IL- 6 and IL-10 secretion in LAB-stimulated PMA-differentiated THP1 macrophages were studied. As shown in Fig. 1, different bacterial species triggered differential cytokines production profiles. Generally, Lactobacillus (L.) plantarum, L. acidophilus and Streptococcus (S.) thermophilus possessed stronger activation potency of both IL-6 and IL-10 production than other species such as L. fermentum, L. casei, L. reuteri, and L. brevis. Moreover, strains within the same species such as L. plantarum also could differentially induce cytokines secretion in immune cells. L. plantarum CCFM634 and L. plantarum CCFM734 initiated higher cytokines production when compared with L. plantarum CCFM 382 L. plantarum CCFM595, and L. plantarum CCFM675.

LPS, a generally accepted pro-inflammatory agent, could barely induce IL-10-production in THP1 macrophages, whereas it could induce a profound IL-6 response. On the contrary, LAB strains such as L. plantarum CCFM634, L. plantarum CCFM734, L. acidophilus CCFM137 and S. thermophilus CCFM218 could dominantly trigger both IL- 6 and IL-10 secretion $(p<0.01, p<0.001)$. Besides, it's worth noting that IL-10 production induced by the latter three strains were much higher than that induced by LPS.

The IL-10 responses here were in the concentration range from 0 to $80 \mathrm{pg} / \mathrm{ml}$. It might be suggested that these are low when compared to other cell sources such as murine bone marrow derived dendritic cells (BMDCs) ${ }^{25}$. However, human macrophage-like THP1 cells ${ }^{36-39}$ but also human primary dendritic cells such as umbilical cord blood-derived $\mathrm{DCs}^{10,11}$ have a IL-10 response in this range ${ }^{10,11,36-39}$.

NF- $\kappa$ B/AP-1 activation induced by different bacterial strains was strain and dose-dependent. Next it was studied whether the species and strain specific effects on cytokine production can be explained by differential effects on pattern recognition receptors (PRRs) activation. To this end, NF- $\kappa \mathrm{B} / \mathrm{AP}-1$ activation induced by bacteria in THP1-XBlue ${ }^{\mathrm{TM}}-\mathrm{MD} 2-\mathrm{CD} 14$ reporter cells was measured. This cell line expresses all TLRs ${ }^{40}$. As shown in Fig. 2, different species of bacteria possessed differential ability to activate NF- $\kappa$ B/AP-1 responses. L. plantarum, L. fermentum, L. acidophilus, S. thermophilus, and L. rhamnosus triggered the highest NF- $\kappa \mathrm{B} /$ AP-1 activation when compared with species such as L. casei, L. reuteri, and L. brevis. Moreover, different strains within the same species could induce NF- $\kappa \mathrm{B} / \mathrm{AP}-1$ responses to different degrees. A representative example is the L. plantarum species: among this species, L. plantarum CCFM634 and L. plantarum CCFM734 activated NF- $\mathrm{B} /$ AP-1 responses, which were statistically significantly higher than the signals induced by L. plantarum CCFM595 and L. plantarum CCFM382 $(p<0.001)$. Some strains such as L. plantarum CCFM675 could barely induce NF- $\kappa \mathrm{B} / \mathrm{AP}-1$ activation.

Furthermore, the NF- $\kappa \mathrm{B} / \mathrm{AP}-1$ induction by the bacteria tested was dose-dependent. It seemed that for most of the strains tested, SEAP secretion was enhanced as the bacteria concentration (bacteria/cells ratios) increased except for L. fermentum CCFM421, which triggered less SEAP production when higher concentration of bacteria were applied.

MyD88-dependency of bacteria-triggered NF- $\kappa$ B signalling. Next, we investigated whether the activation was TLR-dependent by testing the effect of the bacteria on THP-1 reporter cell line pretreated with MyD88 inhibitory peptide Pepinh-MYD. MyD88 is an essential adapter molecule for TLR2, 4, 5, 7, 8, and 9 signalling $^{13}$. Pretreatment with Pepinh-MYD induced a strong and statistically significant reduction in NF- $\kappa$ B/AP-1 responses of most species tested in case of L. plantarum (CCFM634, CCFM382, CCFM734, CCFM595), L. fermentum (CCFM787, CCFM381, CCFM421), L. acidophilus (CCFM137), S. thermophilus (CCFM218), and L. rhamnosus (CCFM237) (Fig. 3a,b). Moreover, MyD88 inhibitor pretreatment seemed to result in the most marked decrease for CCFM787-initiated activation (more than $70 \%$ decrease at the two higher concentrations, $p<0.001$ ), which indicated that in this strain MyD88-dependent pathways predominantly mediated the activation of NF- $\mathrm{KB} / \mathrm{AP}-1$. The strains such as L. casei CCFM30, L. reuteri CCFM14, and L. brevis CCFM498 were not so much inhibited by the MyD88 inhibitor and therefore predominantly activate NF- $\kappa$ B/AP-1 via Myd88-independent pathways. 
IL-6

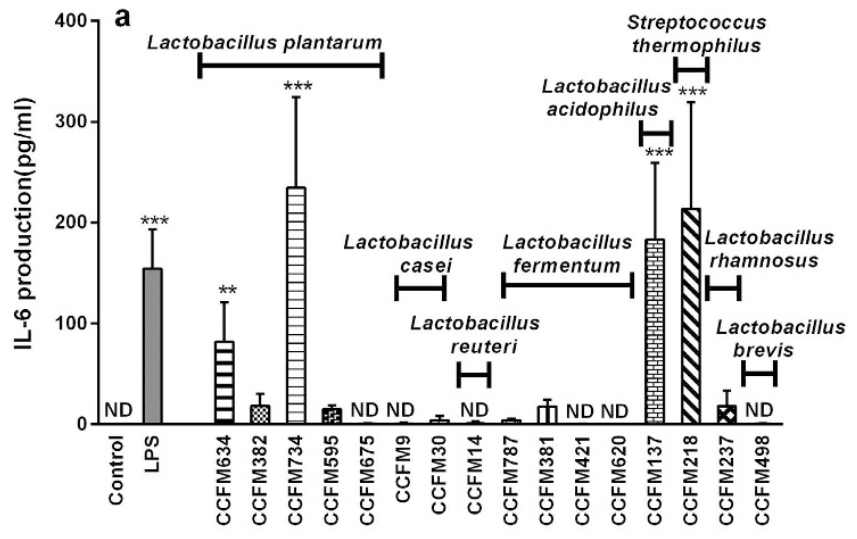

IL-10

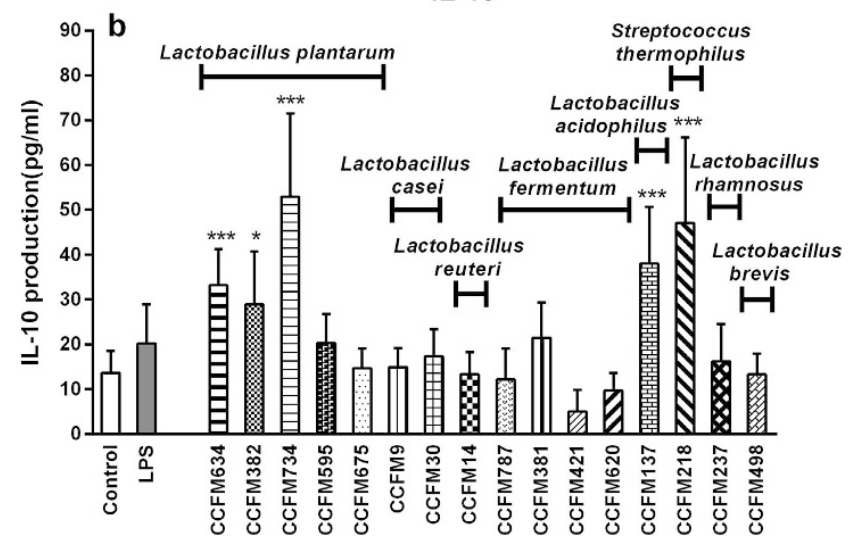

Figure 1. LAB-induced cytokine production in PMA-stimulated THP1 macrophages. PMA-stimulated THP1 macrophages were treated with different bacteria (bacteria to cells ratios of 40:1) or 1 $\mu \mathrm{g} / \mathrm{ml}$ LPS (positive control) for $24 \mathrm{~h}$. Assays were performed in duplicate wells. Untreated THP1 macrophages served as negative control. IL- 6 and IL-10 levels in the supernatant were measured by ELISA. The results shown represent mean and standard deviation (SD) of four independent experiments. ND, not detectable. Statistical significance between different treatment groups and untreated control group was measured using one-way ANOVA with application of the Bonferroni multiple comparisons test $\left({ }^{\star} p<0.05 ;{ }^{* *} p<0.01,{ }^{* *} p<0.001\right)$.

From the above results, we conclude that the selected bacteria might signal through both MyD88-dependent and MyD88-independent PRR signalling pathways, leading to NF- $\kappa \mathrm{B} / \mathrm{AP}-1$ responses.

Bacteria robustly activated TLR2 signalling pathways in a dose-dependent manner. The bacteria that stimulated via TLR-dependent pathways were selected for the next stage in which the specific signalling TLR was identified. To this end HEK reporter cell lines, each carrying one construct for a specific TLR, were stimulated with six selected strains (L. plantarum CCFM634, L. plantarum CCFM734, L. fermentum CCFM787, L. fermentum CCFM381, L. acidophilus CCFM137, and S. thermophilus CCFM218). As shown in Fig. 4, the bacterial strains strikingly activated HEK-Blue ${ }^{\mathrm{TM}}$ hTLR2 cells $(p<0.05, p<0.001)$, and slight activation of TLR7 as well as TLR9 was observed by L. plantarum CCFM634. Furthermore, the response of bacteria to HEK-Blue ${ }^{\text {TM }}$ hTLR2 cells was dose-dependent, which was in accordance with their activation pattern of NF- $\mathrm{B} / \mathrm{AP}-1$ signalling in THP1-XBlue ${ }^{\mathrm{TM}}$ reporter cells.

Bacteria had no dominant inhibitory effect on agonists-induced NF- $\kappa$ B activation. Even though activation of TLR signalling is critical for maintaining health, excessive initiation of TLR signalling will be harmful, which might aggregate several diseases ${ }^{41,42}$. To evaluate the TLR-inhibitory properties of bacteria, we tested the effect of bacteria on agonists-induced TLR activation in HEK-Blue ${ }^{\mathrm{TM}}$ reporter cell lines. However, no remarkable inhibitory effects of agonists-induced TLR activation by various bacteria were found in the HEK-Blue ${ }^{\mathrm{TM}}$ cell lines (Fig. 5, Supplemental Fig. S2). Nevertheless, enhancement of a few pre-existed TLR responses were seen with several strains. All the strains tested could augment TLR2 signalling induced by agonists, which is consistent with their predominant activation of TLR2 when HEK-Blue ${ }^{\mathrm{TM}}$ hTLR2 cells were only treated with bacteria (Figs 4a and 5a). Additionally, L. plantarum CCFM634 elevated agonists-stimulated NF- $\kappa B$ responses in HEK-Blue ${ }^{\mathrm{TM}} \mathrm{hTLR}^{\mathrm{T}}, 9$ cells (Fig. 5c,e), which correlates with its mild activation of TLR7 and TLR9 signalling obtained by co-incubating HEK-Blue ${ }^{\mathrm{TM}}$ hTLR7,9 cells with solely bacteria (Fig. 4b,c). Besides, it is worth noting that some other strains 


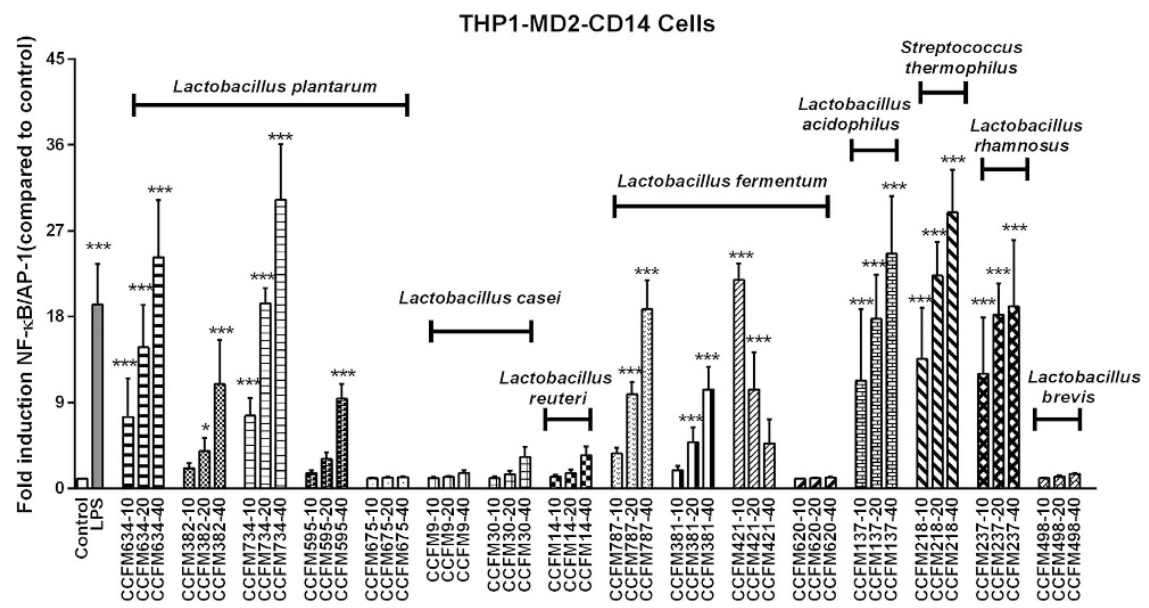

Figure 2. Bacteria-induced differential activation of NF- $\kappa B / A P-1$ in THP1-XBlue ${ }^{\mathrm{TM}}-\mathrm{MD} 2-\mathrm{CD} 14$ cells speciesand dose-dependently. SEAP production in cell culture supernatants was measured to assess NF- $\kappa \mathrm{B} / \mathrm{AP}-1$ activity as described in Materials and Methods section. For each strain, $-10,-20$ and -40 represent different bacteria/ cells ratios respectively. NF- $\kappa \mathrm{B} / \mathrm{AP}-1$ activity is presented as percentage of untreated control cells. The results shown represent mean and standard deviation (SD) of three independent experiments. Statistical significance between different treatment groups and untreated control group was measured using one-way ANOVA with Bonferroni multiple comparisons test $\left({ }^{*} p<0.05 ;{ }^{* *} p<0.01,{ }^{* * *} p<0.001\right)$.

tested (L. plantarum CCFM734, L. fermentum CCFM787, and L. fermentum CCFM381) slightly increased TLR7 signalling when compared with agonist-initiated responses, while when only bacteria were added to HEK-Blue ${ }^{\mathrm{TM}}$ hTLR7 cells no NF- $\kappa B / A P-1$ activity was observed (Figs $4 b$ and $5 c$ ). This might indicate there's a synergistic effect between those bacteria and TLR7 agonist. The similar mild up-regulation of NF- $\kappa \mathrm{B} / \mathrm{AP}-1$ transcription was also observed in HEK-Blue ${ }^{\mathrm{TM}}$ hTLR5, 8 cells (Fig. 5b,d).

Bacteria were predominantly recognized via TLR2/TLR6 heterodimer. For full activation, TLR2 must form heterodimers with TLR1 or TLR6 ${ }^{18}$. As such, in the HEK293 TLR2 reporter cell line, which also constitutively expresses TLR1 and TLR6, TLR2 dimerizes with TLR1 or TLR6 and activates different pathways $^{20,21}$. Several previous studies have validated that TLR2/TLR6 clusters could promote regulatory T (Treg) cells responses, whereas TLR2/TLR1 facilitated pro-inflammatory immune reactions in vivo ${ }^{22-24}$. These earlier findings raise a question as to whether TLR1 or TLR6 was involved in the interaction between TLR2 and bacteria in this study. Therefore, TLR1 or TLR6 neutralizing antibody was used separately with THP1-XBlue ${ }^{\mathrm{TM}}-\mathrm{MD} 2-\mathrm{CD} 14$ cells to block TLR1 or TLR6 signalling. Intriguingly, TLR6 neutralization significantly dampened all the tested strain-induced NF- $\kappa$ B/AP-1 activity (Fig. 6b). On the contrary, TLR1 neutralization appeared to have no effect or even enhanced the signals initiated by some strains (L. plantarum CCFM634, L. plantarum CCFM734, L. fermentum CCFM381, L. acidophilus CCFM137, and S. thermophilus CCFM218) especially in the two lower concentrations (Fig. 6a). But for the strain L. fermentum CCFM787, its activation of NF- $\mathrm{B} / \mathrm{AP}-1$ at three various concentrations were reduced to a lesser magnitude by anti-TLR1 antibody treatment as compared with the effect of anti-TLR6 antibody (Fig. 6a,b). It suggests that both TLR1 and TLR6 were involved in signalling responses induced by $L$. fermentum CCFM787, in which TLR6 prominently mediated its recognition by host immune system. In conclusion, the above observations indicate that TLR2/TLR6 heterodimer were primarily involved in the interactions between the strains tested and host immune cells.

\section{Discussion}

In order to gain insight in the signalling pathways of the LAB strains, we applied a strategic approach. First, THP1 reporter cells expressing all the TLRs were applied for the first screening of LAB strains with PRRs-activating capacities. To this end, 16 LAB strains were selected based on a literature research for strains or parental strains with reported health-promoting functions ${ }^{28-35}$. Profound variations among different species and strains of LAB were found. Next MyD88 dependency was confirmed to demonstrate TLRs involvement. MyD88 inhibitor pretreatment caused a strong decrease in activation but not all strains were influenced illustrating that also other, MyD88-independent PRRs, can be involved in beneficial effects of a number of LAB strains. Next we determined in HEK reporter cell lines the specific TLRs involved in the signalling pathways of selected LAB strains. By having insight in the specific TLR-signalling of LAB we can predict for which application they may be used as will be discussed in the next paragraphs.

Many LAB strains have been reported to possess health-promoting functions in consumers, and are used as food or pharmaceutical probiotic formulations ${ }^{1}$. Despite their application it is still largely unknown how specific LAB strains contribute to health. This study was undertaken to provide more insight in how LAB with confirmed health benefits ${ }^{28-35,43,44}$ might contribute to the beneficial effects. Modulation of the immune system has been extensively proposed as an action of some $\mathrm{LABs}^{1,6,9}$. This can occur via effects on the microbiota ${ }^{6,9}$, but also by direct contact with immune cells in the small intestine ${ }^{6}$. This direct contact is considered to be specific for the small intestine because of differences in mucus composition. The small intestine has a single, loose and permeable 

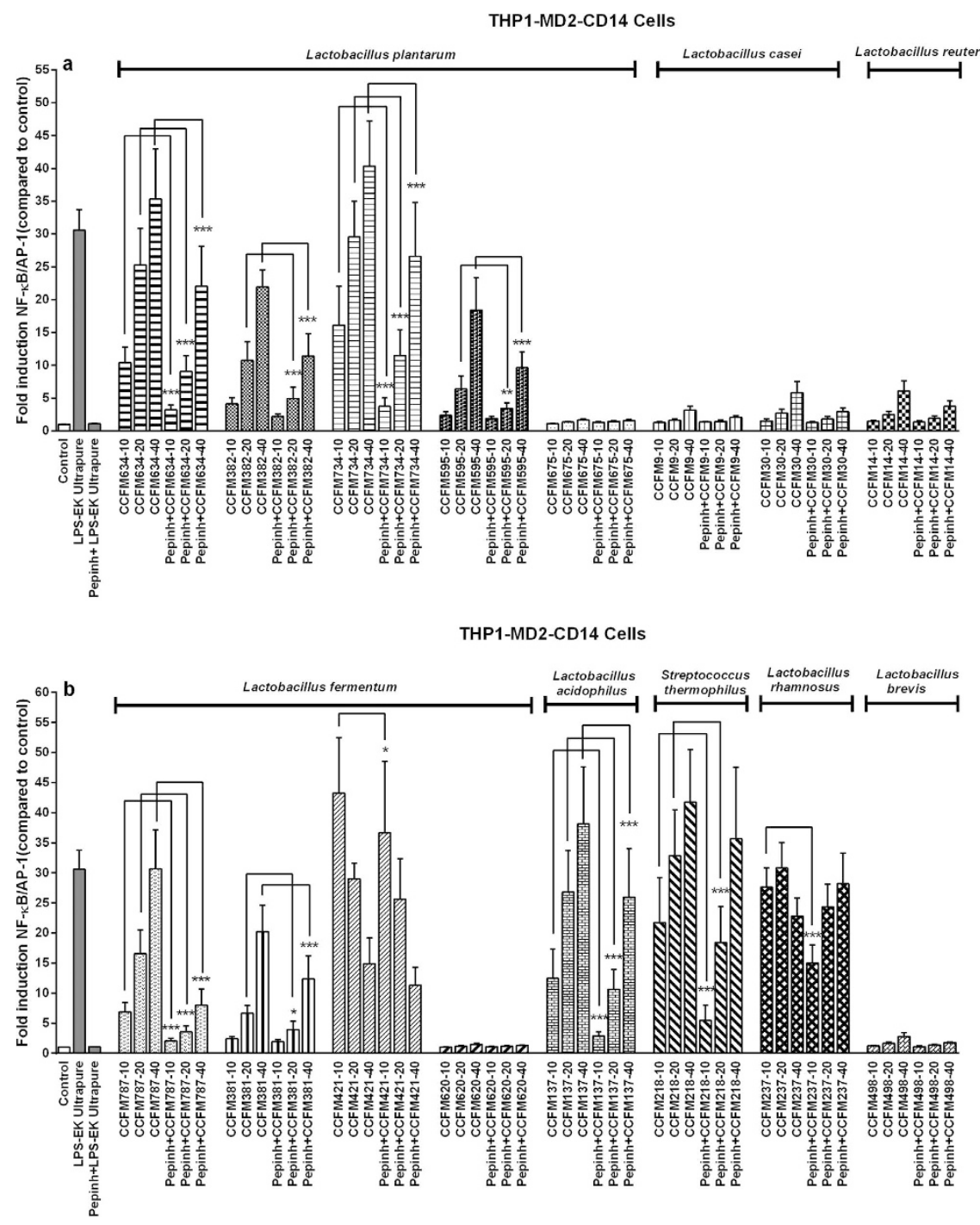

Figure 3. Bacteria signalled via MyD88-dependent pathways. THP1-XBlue ${ }^{\mathrm{TM}}-\mathrm{MD} 2-\mathrm{CD} 14$ cells were pretreated with or without MyD88 inhibitory peptide (Pepinh-MYD) for $6 \mathrm{~h}$ before stimulation with bacteria. After $24 \mathrm{~h}$ of stimulation with bacteria, NF- $\kappa \mathrm{B} / \mathrm{AP}-1$ activity was determined. NF- $\kappa \mathrm{B} / \mathrm{AP}-1$ activity is presented as percentage of un-stimulated control cells. For each strain, $-10,-20$ and -40 represent different bacteria/ cells ratios respectively. The results shown represent mean and standard deviation (SD) of three independent experiments. Statistical significance between inhibitor-treated groups and respective untreated group was measured using two-way ANOVA test with Bonferroni multiple comparisons test $\left({ }^{*} p<0.05 ;{ }^{* *} p<0.01\right.$, $\left.{ }^{* * *} p<0.001\right)$.

mucus layer, which is distinct from the colon ${ }^{45}$. The colon has both a firm inner and an outer loose mucus layer ${ }^{45}$. The loose mucus structure in the small intestine enables direct interactions between LAB and intestinal cells such as epithelium, macrophages, and dendritic cells as previously reported ${ }^{12,46,47}$. Ligation of MAMPs to PRRs in LAB-host crosstalk is proposed to determine the immunomodulating capacity of $\mathrm{LAB}^{12}$. TLRs have been widely studied as one of the receptors for MAMPS on LAB surfaces ${ }^{12,16-18}$. However, as shown here not all LAB strains act via TLRs and many LAB strains may also stimulate other PRRs. This demonstrates that many LAB strains might have TLR independent health benefits. Moreover, our data suggest that every single tested LAB has a unique PRR signalling profile which might be explained by expression of different ligands on the surface of the bacteria or secretion of PRR-stimulating bactericides or other immune active bacterial products ${ }^{48-52}$.

In previous studies we have been researching ligands on probiotic strains responsible for immune modulating effect and identified a few potential new MAMPs for immune stimulation ${ }^{53,54}$. We also showed that the beneficial immune effects of many bacteria were exerted on the host by supporting Treg cell development ${ }^{4,5}$. The generation of Treg cells is highly TLR2/TLR6 dependent and therefore our current experiments were designed to select LABs with Treg supporting effects, i.e. LABs with a TLR2/TLR6 signalling depending effect. As shown in the current study only six of the original 16 strains selected for their health effects ${ }^{28-35}$ act via TLR2/TLR6. This underlines the conclusions of our previous studies demonstrating that selection of specific probiotic strains for enforcing specific desired immune responses may be a mandatory strategy for targeted improvement of host health. The six bacteria 

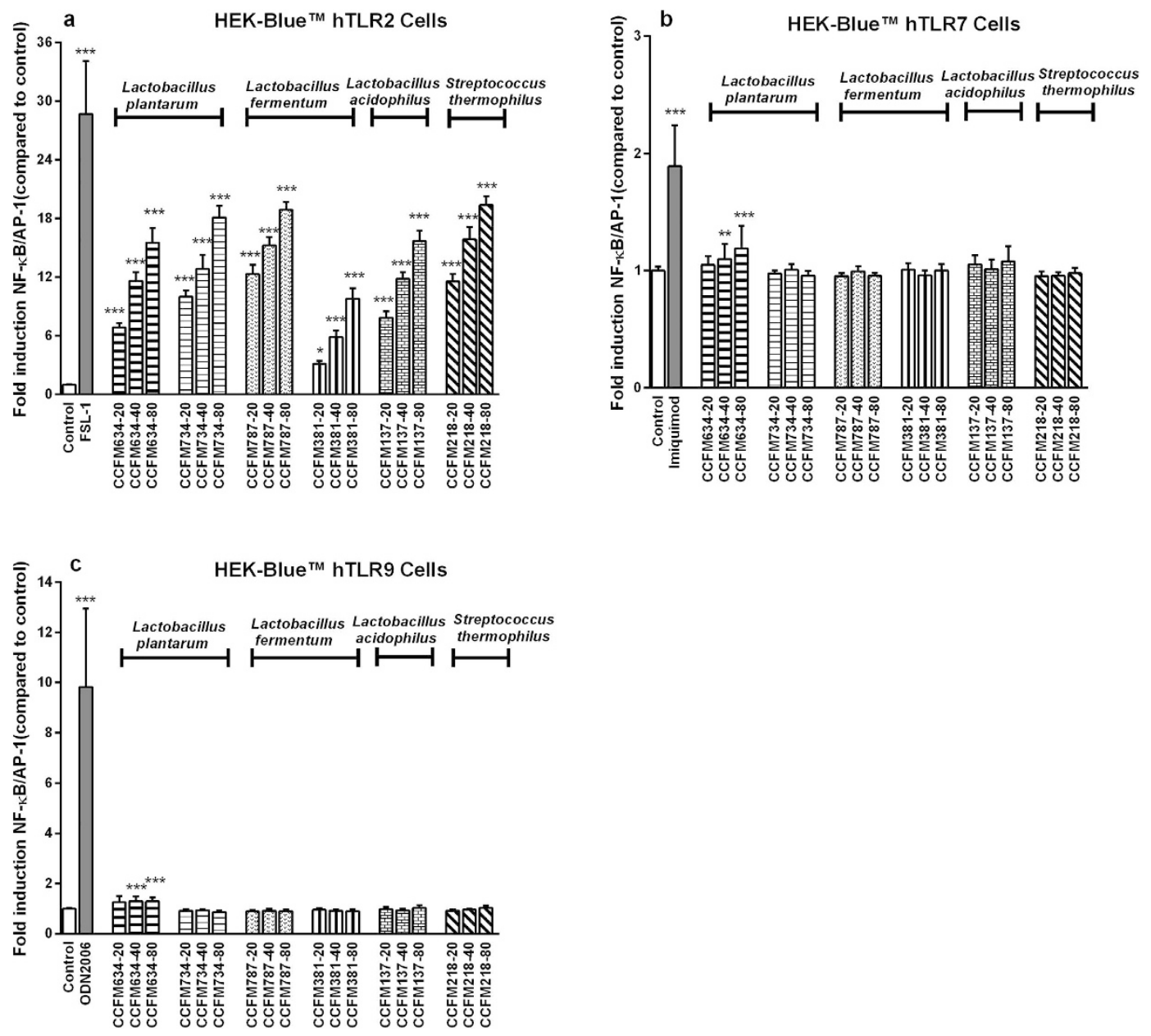

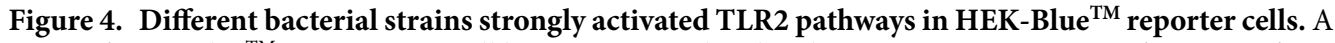
series of HEK-Blue ${ }^{\text {TM }}$ TLR reporter cell lines were stimulated with various concentrations of strains. After $24 \mathrm{~h}$ of co-incubation, SEAP activity in cell culture supernatants was assessed. Respective agonists for TLR served as positive control group. NF- $\kappa \mathrm{B} / \mathrm{AP}-1$ activity is presented as percentage of untreated control cells. For each strain, $-20,-40$ and -80 represent different bacteria/cells ratios respectively. The results shown represent mean and standard deviation (SD) of three independent experiments. Statistical significance between different treatment groups and untreated control group was measured using one-way ANOVA with Bonferroni multiple comparisons test $\left({ }^{*} p<0.05 ;{ }^{* *} p<0.01,{ }^{* * *} p<0.001\right)$.

are of interest as beneficial effects of TLR2-6 signalling have been included in the prevention or management of many diseases such as chronic inflammatory bowel disease and mucositis ${ }^{22,55}$.

In all cases we confirmed the involvement of TLR2/TLR6 by application of antibody blocking, in which neutralization of TLR6 led to attenuation of NF- $\kappa$ B activation of all the six LAB strains in THP1 reporter cell line. Unexpectedly, NF- $\kappa B$ signalling initiated by these strains, except for L. fermentum CCFM787, appeared to be enhanced by blockade of TLR1. The promoting effects of TLR1 neutralization on bacteria-activated NF- $\kappa \mathrm{B} / \mathrm{AP}-1$ responses could be explained as follows. TLR2/TLR1 and TLR2/TLR6 heterodimers are freely spread on the cell surface $^{56}$. TLR1 antibody administration can induce aggregation of TLR2/TLR1 clusters, which would enlarge the surface area on the cell membrane, so that the opportunity of contact and binding between TLR2/TLR6 and microbial ligands would be increased. This would facilitate downstream NF- $\kappa \mathrm{B} / \mathrm{AP}-1$ transcription as previously also reported for PRR activation by particulate $B$-glucan ${ }^{56,57}$.

Intriguingly, in this study we observed that L. fermentum CCFM787 had both TLR2/TLR1 and TLR2/TLR6 signalling capacity since not only TLR6 blockade, but also TLR1 blockade resulted in a (modest) diminished NF- $\kappa$ B/AP-1 activity. This suggests a possible bidirectional immunomodulatory role of this strain, of which anti-inflammatory properties seem to be the dominant action as TLR2/TLR6 was more strongly stimulated. However, since TLR2/TLR1 contributes to proinflammatory responses it is not recommended to use this strain in diseases with ongoing inflammation. This finding demonstrates to our opinion the usefulness of our technology platform for selecting specific strains for TLR management and excluding strains from which undesired effects can be predicted.

To date, most studies have been focusing on the PRR activation profile of bacteria ${ }^{22,46,58-60}$, and not on their inhibitory potentials. Inhibition of TLRs signalling may be of clinical or consumer benefit as in some conditions 

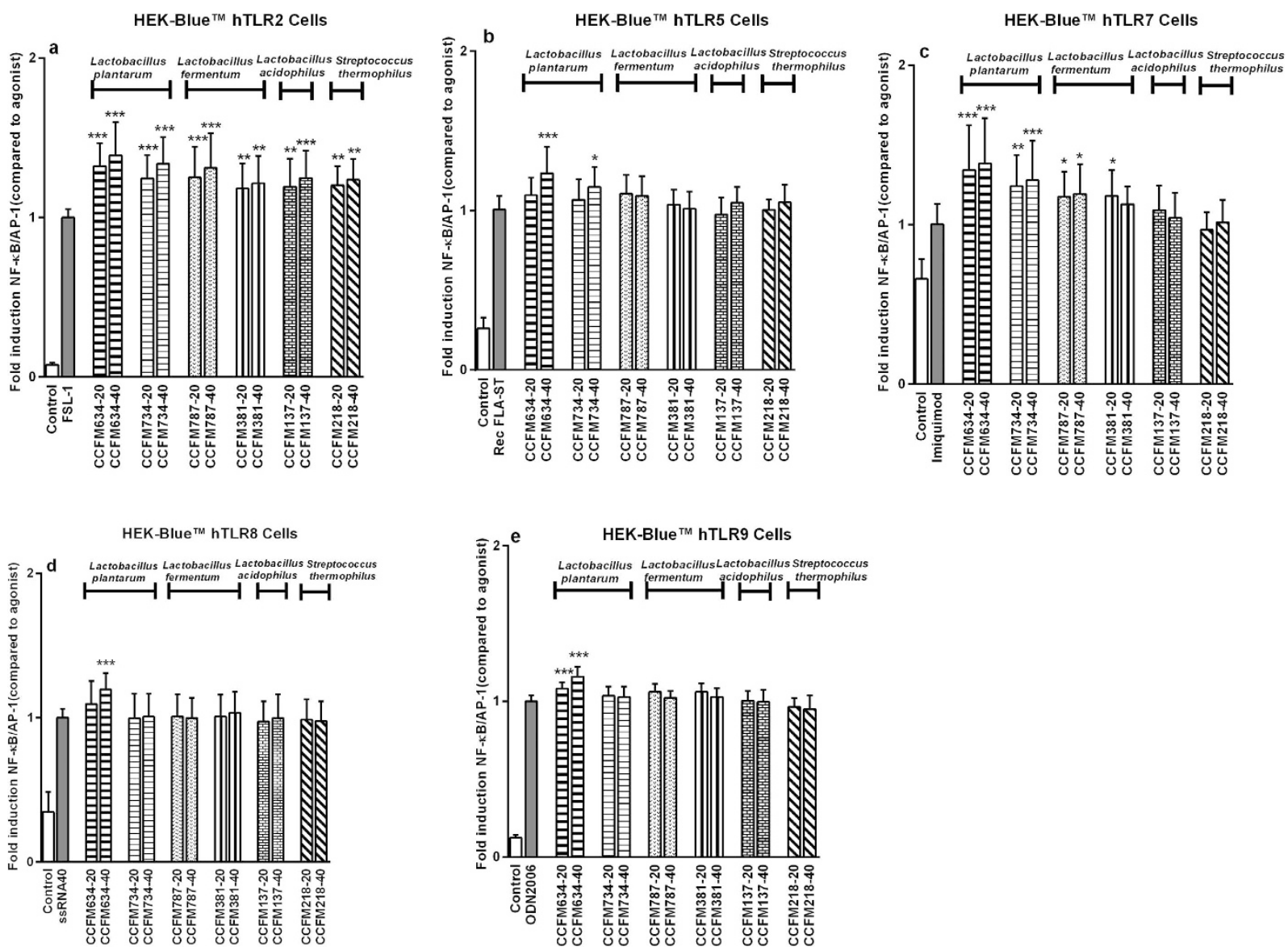

Figure 5. Effect of bacteria on agonists-induced NF- $\kappa B / A P-1$ activation. HEK-Blue ${ }^{\mathrm{TM}}$ TLR reporter cells were stimulated with their agonists and bacteria for $24 \mathrm{~h}$. Then SEAP activity in cell culture supernatants was measured. NF- $\mathrm{B} / \mathrm{AP}-1$ activity is presented as percentage of signals induced by respective agonists. For each strain, -20 and -40 represent different bacteria/cells ratios respectively. The results shown represent mean and standard deviation (SD) of three independent experiments. Statistical significance between different bacteria treatment groups and agonists treatment group was measured using one-way ANOVA with Bonferroni multiple comparisons test $\left({ }^{*} p<0.05 ;{ }^{* *} p<0.01,{ }^{* * *} p<0.001\right)$.

stimulation of TLRs such as TLR4 may lead to enhanced or sustained inflammation ${ }^{61,62}$. To the best of our knowledge we are the first to study inhibition of TLRs by bacteria, but to our surprise, no significant inhibitory effects of the tested LAB strains were observed in this study. What we did observe, however, was synergism in activation between TLR7 agonist and several LAB strains such as L. plantarum CCFM734, L. fermentum CCFM787, and L. fermentum CCFM381. This reveals the existence of synergistic effects of specific LAB strains and ligands of PRRs such as TLR7 agonists which might be an additional mechanism by which bacteria influence health of consumers.

To the best of our knowledge, this is the first study applying a targeted strategy to select LAB strains with specific TLR-dependent signalling capacity. Our screening starts with identifying the possible underlying molecular mechanisms associated with immunomodulatory functions of LAB rather than directly testing in animal- or human-disorders. We feel this is cost- and time-effective as well as selective as we have the opportunity to select the most suitable LAB in a high-throughput fashion.

\section{Material and Methods}

Preparation of bacteria. All bacterial strains used in this study were provided by Culture Collections of Food Microbiology (CCFM), and listed in Table 1. All strains were cultured in De Man-Rogosa-Sharpe (MRS) broth (Merck, Darmstadt, Germany) under aerobic conditions at $37^{\circ} \mathrm{C}$ until stationary phase. Then bacteria were washed twice in phosphate buffered saline (PBS, $\mathrm{pH} 7.4$ ), suspended at appropriate concentrations in PBS containing $20 \%$ glycerol, and stored at $-80^{\circ} \mathrm{C}$ until used. After freezing viable bacterial counts (CFUs) were quantified by plating serial dilutions on MRS agar.

Cell cultures. Human monocytic THP1 cell line was obtained from American Type Culture Collection (ATCC), and was cultured in RPMI 1640 medium (Lonza, Verviers, belgium) with 10\% fetal bovine serum (Sigma-Aldrich, St. Louis, MO USA), $2 \mathrm{mM}$ L-glutamine (Lonza, Verviers, belgium), $1 \mathrm{mM}$ sodium pyruvate (Lonza, Verviers, belgium), $0.05 \mathrm{mM}$ 2-mercaptoethanol (Scharlau, Barcelona, Spain), $60 \mu \mathrm{g} / \mathrm{ml}$ gentamicin 

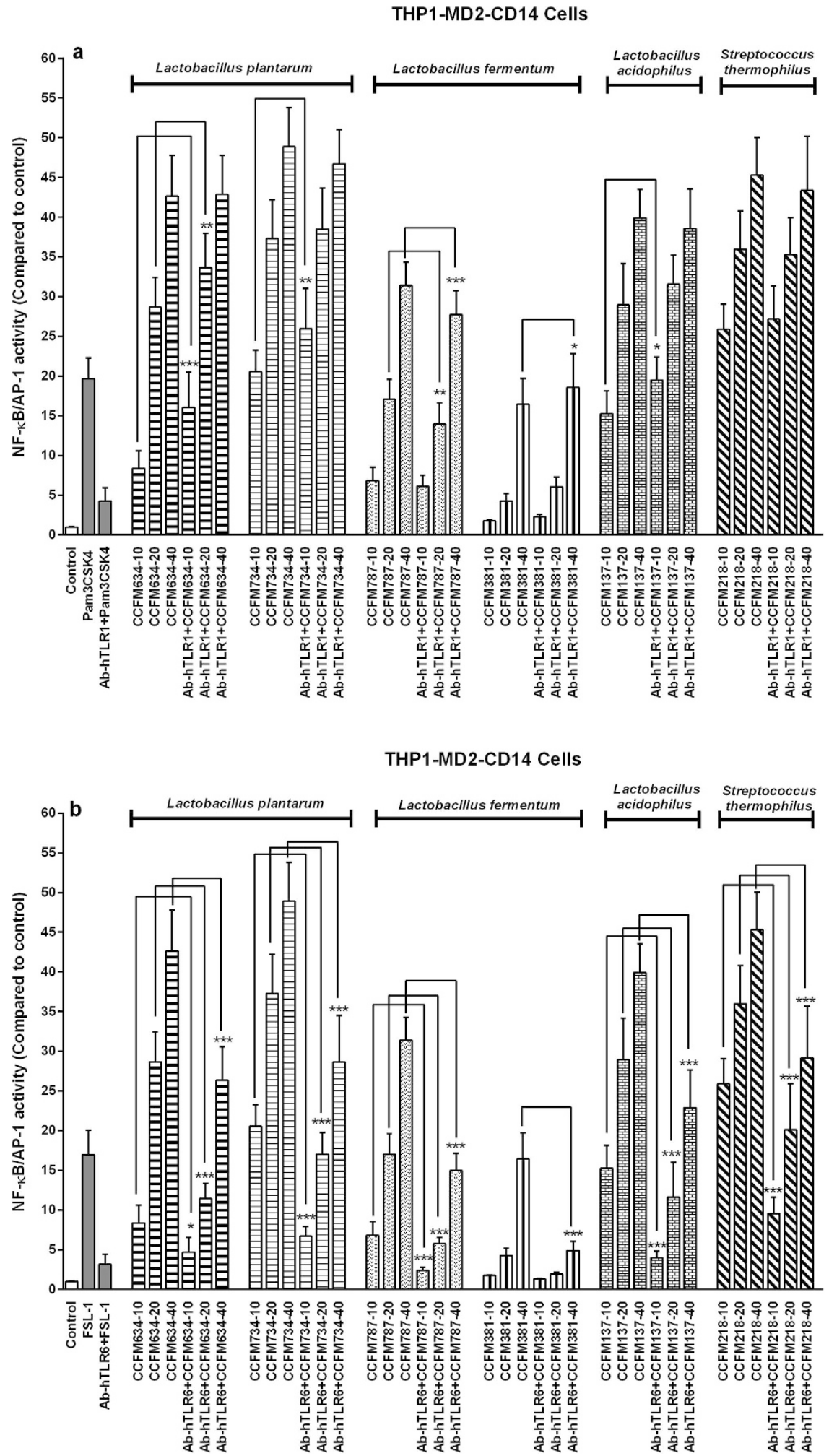

Figure 6. Antibody for TLR1 or TLR6 neutralization assay in THP1-XBlue ${ }^{\mathrm{TM}}-\mathrm{MD} 2-\mathrm{CD} 14$ cells. THP1$\mathrm{XBlue}^{\mathrm{TM}}$-MD2-CD14 cells were pre-treated with or without TLR1 (a) or TLR6 (b) antibody (Ab-hTLR1 or hTLR6; $0.2 \mathrm{mg} / \mathrm{ml}$ ) for $1 \mathrm{~h}$, then bacteria was added to cells. After $24 \mathrm{~h}$ of stimulation, NF- $\mathrm{B} / \mathrm{AP}-1$ activity was determined. NF- $\kappa \mathrm{B} / \mathrm{AP}-1$ activation is presented as percentage of un-stimulated control cells. For each strain, $-10,-20$ and -40 represent different bacteria/cells ratios respectively. The results shown represent mean and standard deviation (SD) of three independent experiments. Statistical significance between antibody-treated groups and respective untreated group was measured using two-way ANOVA test with Bonferroni multiple comparisons test $\left({ }^{*} p<0.05 ;{ }^{* *} p<0.01,{ }^{* * *} p<0.001\right)$. 


\begin{tabular}{|l|l|l|}
\hline Bacterial species & Strain designation & Source or reference \\
\hline Lactobacillus plantarum & CCFM634 & CGMCC9740; Chinese Sichuan pickle isolate \\
\hline Lactobacillus plantarum & CCFM595 & CGMCC9511; Chinese Sichuan pickle isolate \\
\hline Lactobacillus plantarum & CCFM382 & CGMCC9734; Chinese traditional leavened isolate \\
\hline Lactobacillus plantarum & CCFM675 & CGMCC9662; human feces isolate \\
\hline Lactobacillus plantarum & CCFM734 & not available \\
\hline Lactobacillus fermentum & CCFM787 & not available \\
\hline Lactobacillus fermentum & CCFM381 & Chinese traditional leavened isolate \\
\hline Lactobacillus fermentum & CCFM421 & Chinese traditional fermented fish isolate \\
\hline Lactobacillus fermentum & CCFM620 & Chinese traditional fermented green beans isolate \\
\hline Lactobacillus casei & CCFM9 & Pickle isolate \\
\hline Lactobacillus casei & CCFM30 & Cow milk isolate \\
\hline Lactobacillus reuteri & CCFM14 & CICC6226; Yoghurt starter strain \\
\hline Lactobacillus rhamnosus & CCFM237 & CGMCC7317 \\
\hline Lactobacillus acidophilus & CCFM137 & human feces isolate \\
\hline Streptococcus thermophilus & CCFM218 & Kefir isolate \\
\hline Lactobacillus brevis & CCFM498 & Chinese northeast sauerkraut isolate \\
\hline & & \\
\hline
\end{tabular}

Table 1. Bacterial strains used in this study. CCFM refers to Culture Collections of Food Microbiology, Jiangnan University, Wuxi, China. CGMCC refers to China General Microbiological Culture Collection Center, Beijing, China. CICC refers to China Center of Industrial Culture Collection, Beijing, China.

sulfate (Lonza, Verviers, belgium), $2.2 \mu \mathrm{g} / \mathrm{ml}$ amphotericin B solubilized (Sigma-Aldrich, St. Louis, MO USA). THP1-XBlue ${ }^{\mathrm{TM}}-\mathrm{MD} 2-\mathrm{CD} 14$ and HEK-Blue ${ }^{\mathrm{TM}}$ TLR reporter cell lines were purchased from InvivoGen (InvivoGen, Toulouse, France) or home made ${ }^{63,64}$. THP1-XBlue ${ }^{\mathrm{TM}}-\mathrm{MD} 2-\mathrm{CD} 14$ cells are derived from human monocytic THP-1 cell line, and stably express MD2 and CD14 to enhance signalling responses. Each of seven HEK293-Blue ${ }^{\text {TM }}$ TLR cell lines was stably transfected with a specific human TLR gene (hTLR2, hTLR3, hTLR4, hTLR5, hTLR7, hTLR8, hTLR9). Both THP1-XBlue ${ }^{\mathrm{TM}}$ and HEK-Blue ${ }^{\mathrm{TM}}$ cell lines carry NF- $\kappa \mathrm{B}$ - and AP-1-inducible secreted embryonic alkaline phosphatase (SEAP) reporter gene. Once TLRs signalling is initiated, NF- $\kappa B$ and AP- 1 will be activated, which will initiate the secretion of SEAP. Thus, SEAP activity in the cell supernatants can be used to quantify NF- $\kappa$ B activation. THP1-XBlue ${ }^{\mathrm{TM}}$ and HEK-Blue ${ }^{\mathrm{TM}}$ cells were cultured as previously described ${ }^{64}$.

THP1 monocytes to macrophage differentiations and stimulations. THP1 monocytes $\left(1 \times 10^{6} \mathrm{cells} / \mathrm{ml}\right)$ were differentiated with $100 \mathrm{ng} / \mathrm{ml}$ Phorbol 12-myristate 13-acetate (PMA, Sigma-Aldrich) in T75 cell culture flask (Corning, New York, USA) for $48 \mathrm{~h}$. Then PMA-differentiated THP1 macrophages were detached and resuspended in fresh culture medium at $1 \times 10^{6}$ cells $/ \mathrm{ml}$. $0.5 \mathrm{ml}$ of cell suspension were seeded per well in 24 -well plates (Corning, New York, USA) and cultured for another $24 \mathrm{~h}$. Subsequently, culture medium were discarded and fresh medium containing various bacterial strains (bacteria to cells ratios of 40:1) or $1 \mu \mathrm{g} / \mathrm{ml}$ LPS (positive control; Invivogen) were added to cells. Un-stimulated cells served as negative control. After $24 \mathrm{~h}$ of stimulation, pro- and anti-inflammatory cytokines (IL-6 and IL-10) secretion in the supernatant were measured by ELISA (eBioscience, San Diego, USA) according to manufacturer's protocol.

Pattern recognition receptor activation by bacteria in reporter cells. Activation of PRR signalling by various bacterial strains was assessed in THP1-XBlue ${ }^{\mathrm{TM}}$ and HEK-Blue ${ }^{\mathrm{TM}}$ cells following the manufacturer's protocol. THP1 and HEK cells were collected and resuspended in fresh culture medium at appropriate cell density (Table 2). Then $100 \mu \mathrm{l}$ of cell suspension per well was seeded in flat-bottom 96 -well plates. For each bacterial strain, initial bacterial suspension stocks were diluted to various concentrations. Cells were stimulated with $10 \mu \mathrm{l}$ of diluted bacteria suspensions (bacteria to cells ratios of 10:1, 20:1, 40:1), respective agonists (positive control; Invivogen) or PBS containing $20 \%$ glycerol (negative control) for $24 \mathrm{~h}$. Then secretion of SEAP in cell supernatants was measured by QUANTI-Blue ${ }^{\mathrm{TM}}$ reagent (InvivoGen, Toulouse, France). The types and concentrations of agonists used were listed in Table 2.

Effect of bacteria on agonists-induced NF- $\kappa$ B activation in HEK-BlueTM cell lines. To investigate the modulatory effect of bacteria on agonists-triggered activation of specific TLR signalling, HEK-Blue ${ }^{\mathrm{TM}}$ reporter cells were co-incubated with the corresponding agonist together with different bacteria. HEK-Blue ${ }^{\mathrm{TM}}$ reporter cells were seeded at adequate concentrations as indicated in Table 2, using $100 \mu \mathrm{l}$ of cell suspension for each well. Then appropriate agonists were added to cells, followed by adding diluted different concentrations of bacterial suspensions (bacteria to cells ratios of 10:1, 20:1, 40:1) or PBS containing 20\% glycerol (served as negative control for bacteria stimulation group). After $24 \mathrm{~h}$ of co-stimulation, SEAP activity in supernatants was determined using QUANTI-Blue $^{\mathrm{TM}}$ reagent (InvivoGen, Toulouse, France) according to the manufacturer's instructions. The concentrations of agonists used were indicated in Table 2.

MyD88 inhibition assay. MyD88 Inhibitory Peptide Pepinh-MYD (InvivoGen) was used to study whether NF- $\kappa B$ activation by bacteria was MyD88-dependent. THP1-XBlue ${ }^{\mathrm{TM}}$ cells were pre-treated with $5 \mu \mathrm{l}$ of MyD88 


\begin{tabular}{|c|c|c|c|}
\hline Cell line & Cell density & $\begin{array}{l}\text { Specific agonist applied as } \\
\text { positive control }\end{array}$ & $\begin{array}{c}\text { Concentration of } \\
\text { agonist added to wells }\end{array}$ \\
\hline THP1-XBlue ${ }^{\mathrm{TM}}$-MD2-CD14 & $1 \times 10^{6} \mathrm{cells} / \mathrm{ml}$ & LPS-EK Ultrapure & $1 \mu \mathrm{g} / \mathrm{ml}$ \\
\hline HEK-Blue ${ }^{\text {TM }}$ hTLR2 & $2.8 \times 10^{5}$ cells $/ \mathrm{ml}$ & FSL-1 & $50 \mathrm{ng} / \mathrm{ml}$ \\
\hline HEK-Blue ${ }^{\text {TM }}$ hTLR3 & $2.8 \times 10^{5}$ cells $/ \mathrm{ml}$ & Poly(I:C) LMW & $1 \mu \mathrm{g} / \mathrm{ml}$ \\
\hline HEK-Blue ${ }^{\mathrm{TM}}$ hTLR4 & $1.4 \times 10^{5} \mathrm{cells} / \mathrm{ml}$ & LPS-EK Ultrapure & $1 \mu \mathrm{g} / \mathrm{ml}$ \\
\hline HEK-Blue ${ }^{\mathrm{TM}}$ hTLR5 & $1.4 \times 10^{5}$ cells $/ \mathrm{ml}$ & Rec FLA-ST & $0.1 \mu \mathrm{g} / \mathrm{ml}$ \\
\hline HEK-Blue ${ }^{\mathrm{TM}}$ hTLR7 & $2.25 \times 10^{5}$ cells $/ \mathrm{ml}$ & Imiquimod (R837) & $100 \mu \mathrm{g} / \mathrm{ml}$ \\
\hline HEK-Blue ${ }^{\mathrm{TM}}$ hTLR8 & $2.25 \times 10^{5}$ cells $/ \mathrm{ml}$ & ssRNA40/LyoVec $^{\text {TM }}$ & $50 \mu \mathrm{g} / \mathrm{ml}$ \\
\hline HEK-Blue ${ }^{\text {TM }}$ hTLR9 & $4.5 \times 10^{5}$ cells $/ \mathrm{ml}$ & ODN 2006 & $2.5 \mu \mathrm{M}$ \\
\hline
\end{tabular}

Table 2. Agonists used for THP1-XBlue ${ }^{\mathrm{TM}}$ and HEK-Blue ${ }^{\mathrm{TM}}$ reporter cell stimulation assay.

inhibitor ( $1 \mathrm{mM}$ ) for $6 \mathrm{~h}$ before stimulation with bacteria. After $24 \mathrm{~h}$ of stimulation with bacteria (bacteria to cells ratios of 10:1, 20:1, 40:1), SEAP activity in cell culture supernatants was quantified as described above for PRR activation assay.

Antibody neutralization assay. The role of specific TLR (TLR1 and TLR6) for bacteria-initiated NF- $\kappa B$ signalling were proven by using polyclonal antibodies specific for human TLR1 and TLR6 (InvivoGen). After seeding THP1-XBlue ${ }^{\mathrm{TM}}$ cells in plates, $10 \mu \mathrm{l}$ of specific anti-hTLR1 or anti-hTLR6 $(0.2 \mathrm{mg} / \mathrm{ml})$ was added to cells and pre-incubated for $1 \mathrm{~h}$ to inhibit the activity of specific TLR. Then cells were co-incubated with bacteria or respective agonists for specific TLR for $24 \mathrm{~h}$. Other procedures were processed the same as in PRR activation assay.

Statistical analysis. Parametric distribution of data points was confirmed using the Kolmogorov-Smirnov test. Statistical comparisons were performed using one-way ANOVA and two-way ANOVA with Bonferroni multiple comparisons test for post-hoc comparison. GraphPad Prism version 6.0 (San Diego, CA, USA) was used to perform statistical tests. Values of $\mathrm{p}<0.05$ were considered to be statistically significant. Data are presented as mean \pm SD. Significant differences were indicated by asterisks: ${ }^{\star} p<0.05 ;{ }^{* *} p<0.01 ;{ }^{* *} p<0.001$.

\section{References}

1. Naidu, A. S., Bidlack, W. R. \& Clemens, R. A. Probiotic spectra of lactic acid bacteria (LAB). Crit Rev Food Sci Nutr. 39, 13-126 (1999).

2. Makarova, K. et al. Comparative genomics of the lactic acid bacteria. Proc Natl Acad Sci USA 103, 15611-15616 (2006).

3. Gilliland, S. E. Health and nutritional benefits from lactic acid bacteria. FEMS Microbiol Lett. 87, 175-188 (1990).

4. Smelt, M. J. et al. L. plantarum, L. salivarius, and L. lactis attenuate Th2 responses and increase Treg frequencies in healthy mice in a strain dependent manner. PLoS One 7, e47244 (2012).

5. Smelt, M. J. et al. Probiotics can generate FoxP3 T-Cell responses in the small intestine and simultaneously inducing CD4 and CD8 T cell activation in the large Intestine. PLoS One 8, e68952 (2013).

6. Bron, P. A., van Baarlen, P. \& Kleerebezem, M. Emerging molecular insights into the interaction between probiotics and the host intestinal mucosa. Nat Rev Micro. 10, 66-78 (2012).

7. Kwon, H.-K. et al. Generation of regulatory dendritic cells and CD4+Foxp3+ T cells by probiotics administration suppresses immune disorders. Proc Natl Acad Sci USA 107, 2159-2164 (2010).

8. Resta-Lenert, S. C. \& Barrett, K. E. Modulation of intestinal barrier properties by probiotics: role in reversing colitis. Ann N Y Acad Sci. 1165, 175-182 (2009).

9. Bermudez-Brito, M., Plaza-Díaz, J., Muñoz-Quezada, S., Gómez-Llorente, C. \& Gil, A. Probiotic mechanisms of action. Ann Nutr Metab. 61, 160-174 (2012).

10. Bermudez-Brito, M. et al. The impact of dietary fibers on dendritic cell responses in vitro is dependent on the differential effects of the fibers on intestinal epithelial cells. Mol Nutr Food Res. 59, 698-710 (2015).

11. Bermudez-Brito, M., Rösch, C., Schols, H. A., Faas, M. M. \& de Vos, P. Resistant starches differentially stimulate toll-like receptors and attenuate proinflammatory cytokines in dendritic cells by modulation of intestinal epithelial cells. Mol Nutr Food Res. 59, 1814-1826 (2015).

12. Lebeer, S., Vanderleyden, J. \& De Keersmaecker, S. C. J. Host interactions of probiotic bacterial surface molecules: comparison with commensals and pathogens. Nat Rev Micro. 8, 171-184 (2010).

13. Takeda, K. \& Akira, S. TLR signaling pathways. Semin Immunol. 16, 3-9 (2004).

14. Liu, H., Komai-Koma, M., Xu, D. \& Liew, F. Y. Toll-like receptor 2 signaling modulates the functions of CD4+CD25+ regulatory T cells. Proc Natl Acad Sci USA 103, 7048-7053 (2006).

15. Podolsky, D. K., Gerken, G., Eyking, A. \& Cario, E. Colitis-associated variant of TLR2 causes impaired mucosal repair because of TFF3 deficiency. Gastroenterology 137, 209-220 (2009).

16. Cario, E., Gerken, G. \& Podolsky, D. K. Toll-like receptor 2 controls mucosal inflammation by regulating epithelial barrier function. Gastroenterology 132, 1359-1374 (2007).

17. Cario, E., Gerken, G. \& Podolsky, D. K. Toll-like receptor 2 enhances ZO-1-associated intestinal epithelial barrier integrity via protein kinase C. Gastroenterology 127, 224-238 (2004).

18. van Bergenhenegouwen, J. et al. TLR 2 \& Co: a critical analysis of the complex interactions between TLR 2 and coreceptors. J Leukoc Biol. 94, 885-902 (2013).

19. Liu, Y., Yin, H., Zhao, M. \& Lu, Q. TLR2 and TLR4 in autoimmune diseases: a comprehensive review. Clin Rev Allergy Immunol. 47, 136-147 (2013).

20. Ozinsky, A. et al. The repertoire for pattern recognition of pathogens by the innate immune system is defined by cooperation between toll-like receptors. Proc Natl Acad Sci USA 97, 13766-13771 (2000).

21. Schröder, N. W. J. et al. Lipopolysaccharide binding protein binds to triacylated and diacylated lipopeptides and mediates innate immune responses. J Immunol. 173, 2683-2691 (2004).

22. DePaolo, R. W. et al. Toll-like receptor 6 drives differentiation of tolerogenic dendritic cells and contributes to LcrV-mediated plague pathogenesis. Cell Host Microbe. 4, 350-361 (2008). 
23. DePaolo, R. W. et al. A specific role for TLR1 in protective TH17 immunity during mucosal infection. J Exp Med. 209, 1437-1444 (2012).

24. Netea, M. G., Van De Veerdonk, F., Verschueren, I., Van Der Meer, J. W. M. \& Kullberg, B. J. Role of TLR1 and TLR6 in the host defense against disseminated candidiasis. FEMS Immunol Med Microbiol 52, 118-123 (2008).

25. Smelt, M. J. et al. The impact of Lactobacillus plantarum WCFS1 teichoic acid D-alanylation on the generation of effector and regulatory T-cells in healthy mice. PLoS One 8, e63099 (2013).

26. Grangette, C. et al. Enhanced antiinflammatory capacity of a Lactobacillus plantarum mutant synthesizing modified teichoic acids. Proc Natl Acad Sci USA 102, 10321-10326 (2005).

27. Claes, I. J. J. et al. Impact of lipoteichoic acid modification on the performance of the probiotic Lactobacillus rhamnosus GG in experimental colitis. Clin Exp Immunol. 162, 306-314 (2010)

28. Karimi, K., Inman, M. D., Bienenstock, J. \& Forsythe, P. Lactobacillus reuteri-induced regulatory T cells protect against an allergic airway response in mice. Am J Respir Crit Care Med. 179, 186-193 (2009).

29. Karczewski, J. et al. Regulation of human epithelial tight junction proteins by Lactobacillus plantarum in vivo and protective effects on the epithelial barrier. Am J Physiol Gastrointest Liver Physiol. 298, G851-G859 (2010).

30. Geier, M. S., Butler, R. N., Giffard, P. M. \& Howarth, G. S. Lactobacillus fermentum BR11, a potential new probiotic, alleviates symptoms of colitis induced by dextran sulfate sodium (DSS) in rats. Int J Food Microbiol. 114, 267-274 (2007)

31. Guérin-Danan, C. et al. Food supplementation with milk fermented by Lactobacillus casei DN-114 001 protects suckling rats from rotavirus-associated diarrhea. J Nutr. 131, 111-117 (2001).

32. Shu, Q. \& Gill, H. S. Immune protection mediated by the probiotic Lactobacillus rhamnosus HN001 (DR20 ${ }^{\mathrm{TM}}$ ) against Escherichia coli O157:H7 infection in mice. FEMS Immunol Med Microbiol. 34, 59-64 (2002).

33. Chen, C.-C., Louie, S., Shi, H. N. \& Walker, W. A. Preinoculation with the probiotic Lactobacillus acidophilus early in life effectively inhibits murine Citrobacter rodentium colitis. Pediatr Res. 58, 1185-1191 (2005).

34. Ueno, N. et al. Heat-killed body of Lactobacillus brevis SBC8803 ameliorates intestinal injury in a murine model of colitis by enhancing the intestinal barrier function. Inflamm Bowel Dis. 17, 2235-2250 (2011).

35. Tooley, K. L., Howarth, G. S., Lymn, K. A., Lawrence, A. \& Butler, R. N. Oral ingestion of Streptococcus thermophilus diminishes severity of small intestinal mucositis in methotrexate treated rats. Cancer Biol Ther. 5, 593-600 (2006).

36. Neumeier, M. et al. Different effects of adiponectin isoforms in human monocytic cells. J Leukocyte Biol. 79, 803-808 (2006).

37. Azuma, Y. \& Ohura, K. Endomorphins 1 and 2 inhibit IL-10 and IL-12 production and innate immune functions, and potentiate NF-kappa B DNA binding in THP-1 differentiated to macrophage-like cells. Scand J Immunol. 56, 260-269 (2002).

38. Essafi-Benkhadir, K. et al. Quince (Cydonia oblonga Miller) peel polyphenols modulate LPS-induced inflammation in human THP-1derived macrophages through NF-kB, p38MAPK and Akt inhibition. Biochem Biophys Res Commun. 418, 180-185 (2012).

39. Ma, W. et al. The $\mathrm{p} 38$ mitogen-activated kinase pathway regulates the human interleukin-10 promoter via the activation of Sp1 transcription factor in lipopolysaccharide-stimulated human macrophages. J Biol Chem. 276, 13664-13674 (2001).

40. Needham, B. D. et al. Modulating the innate immune response by combinatorial engineering of endotoxin. Proc Natl Acad Sci USA 110, 1464-1469 (2013)

41. Abreu, M. T., Fukata, M. \& Arditi, M. TLR Signalling in the gut in health and disease. J Immunol. 174, 4453-4460 (2005).

42. Cook, D. N., Pisetsky, D. S. \& Schwartz, D. A. Toll-like receptors in the pathogenesis of human disease. Nat Immunol. 5, 975-979 (2004).

43. Wagner, R. D. et al. Biotherapeutic effects of probiotic bacteria on candidiasis in immunodeficient mice. Infect Immun. 65, 4165-4172 (1997).

44. Roselli, M. et al. Prevention of TNBS-induced colitis by different Lactobacillus and Bifidobacterium strains is associated with an expansion of $\gamma \delta \mathrm{T}$ and regulatory $\mathrm{T}$ cells of intestinal intraepithelial lymphocytes. Inflamm Bowel Dis. 15, 1526-1536 (2009).

45. Hansson, G. C. Role of mucus layers in gut infection and inflammation. Curr Opin Microbiol. 15, 57-62 (2012).

46. Winkler, P., Ghadimi, D., Schrezenmeir, J. \& Kraehenbuhl, J.-P. Molecular and cellular basis of microflora-host interactions. J Nutr. 137, 756S-772S (2007).

47. Wells, J. M., Rossi, O., Meijerink, M. \& van Baarlen, P. Epithelial crosstalk at the microbiota-mucosal interface. Proc Natl Acad Sci USA 108, 4607-4614 (2011).

48. Tang, Y. et al. Exopolysaccharide produced by Lactobacillus plantarum induces maturation of dendritic cells in BALB/c mice. PLoS One 10, e0143743 (2015).

49. Bermudez-Brito, M., Muñoz-Quezada, S., Gomez-Llorente, C., Romero, F. \& Gil, A. Lactobacillus rhamnosus and its cell-free culture supernatant differentially modulate inflammatory biomarkers in Escherichia coli-challenged human dendritic cells. Br J Nutr. 111, 1727-1737 (2014).

50. Harb, H. et al. Neonatal supplementation of processed supernatant from Lactobacillus rhamnosus GG improves allergic airway inflammation in mice later in life. Clin Exp Allergy 43, 353-364 (2013).

51. Thomas, C. M. et al. Histamine derived from probiotic Lactobacillus reuteri suppresses TNF via modulation of PKA and ERK signaling. PLoS One 7, e31951 (2012).

52. Vincenti, J. E. The influence of cell-free Lactobacillus rhamnosus GG supernatant on the phagocytic activity of macrophages. Bioscience Horizons 3, 105-112 (2010).

53. van Hemert, S. et al. Identification of Lactobacillus plantarum genes modulating the cytokine response of human peripheral blood mononuclear cells. BMC Microbiol. 10, 1-13 (2010).

54. Meijerink, M. et al. Identification of genetic loci in Lactobacillus plantarum that modulate the immune response of dendritic cells using comparative genome hybridization. PLoS One 5, e10632 (2010).

55. Sultani, M., Stringer, A. M., Bowen, J. M. \& Gibson, R. J. Anti-Inflammatory cytokines: important immunoregulatory factors contributing to chemotherapy-induced gastrointestinal mucositis. Chemother Res Pract. 2012, 11 (2012).

56. Triantafilou, M. et al. Membrane sorting of toll-like receptor (TLR)-2/6 and TLR2/1 heterodimers at the cell surface determines heterotypic associations with CD36 and intracellular targeting. J Biol Chem. 281, 31002-31011 (2006).

57. Sahasrabudhe, N. M. et al. Endo-glucanase digestion of oat $\beta$-glucan enhances Dectin-1 activation in human dendritic cells. J Funct Foods. 21, 104-112 (2016).

58. Kaji, R., Kiyoshima-Shibata, J., Nagaoka, M., Nanno, M. \& Shida, K. Bacterial teichoic acids reverse predominant IL-12 production induced by certain Lactobacillus strains into predominant IL-10 production via TLR2-dependent ERK activation in macrophages. J Immunol. 184, 3505-3513 (2010).

59. Vizoso Pinto, M. G. et al. Lactobacilli stimulate the innate immune response and modulate the TLR expression of HT29 intestinal epithelial cells in vitro. Int J Food Microbiol. 133, 86-93 (2009).

60. Kim, Y.-G. et al. Probiotic Lactobacillus casei activates innate immunity via NF- $\mathrm{B}$ and p38 MAP kinase signaling pathways. Microbes Infect. 8, 994-1005 (2006).

61. Eisenbarth, S. C. et al. Lipopolysaccharide-enhanced, toll-like receptor 4-dependent T helper cell type 2 responses to inhaled antigen. J Exp Med. 196, 1645-1651 (2002).

62. Liew, F. Y., Xu, D., Brint, E. K. \& O’Neill, L. A. J. Negative regulation of toll-like receptor-mediated immune responses. Nat Rev Immunol. 5, 446-458 (2005).

63. Vogt, L. et al. Immune modulation by different types of $\beta 2 \rightarrow 1$-fructans is toll-like receptor dependent. PLoS One 8, e68367 (2013).

64. Paredes-Juarez, G. A., de Haan, B. J., Faas, M. M. \& de Vos, P. The role of pathogen-associated molecular patterns in inflammatory responses against alginate based microcapsules. J Control Release 172, 983-992 (2013). 


\section{Acknowledgements}

The study was financially supported by the China Scholarship Council (CSC). The studies were performed within the framework of the Carbohydrate Competence Center. This center has been financially supported by the European Union, the European Regional Development Fund, and The Northern Netherlands Provinces (Samenwerkingsverband Noord-Nederland), KOERS NOORD.

\section{Author Contributions}

C.R. and P.d.V. conceived and designed the experiments. C.R. performed the experiments and analyzed data. C.R., Q.Z., B.J.d.H., H.Z., M.M.F. and P.d.V. wrote the paper.

\section{Additional Information}

Supplementary information accompanies this paper at http://www.nature.com/srep

Competing financial interests: The authors declare no competing financial interests.

How to cite this article: Ren, C. et al. Identification of TLR2/TLR6 signalling lactic acid bacteria for supporting immune regulation. Sci. Rep. 6, 34561; doi: 10.1038/srep34561 (2016).

(c) (i) This work is licensed under a Creative Commons Attribution 4.0 International License. The images or other third party material in this article are included in the article's Creative Commons license, unless indicated otherwise in the credit line; if the material is not included under the Creative Commons license, users will need to obtain permission from the license holder to reproduce the material. To view a copy of this license, visit http://creativecommons.org/licenses/by/4.0/

(C) The Author(s) 2016 